\title{
Resonance Magnetoresistance in Coupled Quantum Wells
}

\author{
Y. Berk ${ }^{1}$, A. Kamenev ${ }^{2}$, A. Palevski ${ }^{1}$, L. N. Pfeiffer ${ }^{3}$, and K. W. West ${ }^{3}$ \\ ${ }^{1}$ School of Physics and Astronomy, Raymond and Beverly Sackler Faculty of Exact Sciences, \\ Tel Aviv University, Tel Aviv 69978, Israel \\ ${ }^{2}$ Department of Condensed Matter, The Weizmann Institute of Science, Rehovot 76100, Israel. \\ 3 ATET Bell Laboratories, Murray Hill, New Jersey 07974
}

(May 6, 2019)

\begin{abstract}
The in-plane magnetic field suppresses the quantum coupling between electrons in a double quantum well structure. The microscopical theory of this effect is developed and confirmed experimentally. We have shown that the decrease of the "resistance resonance" peak is sensitive to the mutual orientation of the current and the in-plane magnetic field. The characteristic field required for the suppression of the resonance depends on the elastic small angle and electron-electron scattering rates. The study of the characteristic field allows to verify the temperature and Fermi energy dependence of the electron-electron scattering rate, providing a new experimental tool for its measurement.
\end{abstract}

PACS. 72.80.Ey, 73.20.Dx, 73.20.Jc

Typeset using REVTEX 
A new physical phenomena, called resistance resonance (RR), in a double quantum well (QW) structure was recently predicted and observed experimentally [1]. The key point of this effect is the following. Let us consider two tunneling coupled QWs. The quantity of interest is the lateral resistance of the structure (all the electrodes are attached to the both QWs !). If the tunneling between QWs is by some reason suppressed, each electron is localized in one of the wells. The resulting lateral resistance is those one of two conductors connected in parallel, $R_{o f f} \sim\left(\tau_{1}^{t r}+\tau_{2}^{t r}\right)^{-1}$, where $\tau_{i}^{t r}$ is the transport mean free time in the $i^{t h}$ well. In the presence of tunneling, the eigenfunctions form symmetric and antisymmetric subbands, leading to delocalization of electrons between two wells. The corresponding scattering rate in each of these subbands is $\left(\tau^{t r}\right)^{-1}=\left(2 \tau_{1}^{t r}\right)^{-1}+\left(2 \tau_{2}^{t r}\right)^{-1}$ and the resistance is given by $R_{\text {res }} \sim\left(2 \tau^{t r}\right)^{-1}$. One notices that if mobilities of the two QWs are different $\left(\tau_{1}^{t r} \neq \tau_{2}^{t r}\right)$, than $R_{\text {res }}>R_{\text {off }}$. The reason is very simple: in the first case (no coupling) the clean well shunts the dirty one, making the resistance small. No such shunting occurs for the coupled wells.

The experimental realization of this idea [1] 3] was based on the displacement of the QW's energy levels by the gate voltage. The typical graph (lateral resistance vs. gate voltage) is plotted on the insert to Fig. 2. The resonance occurs in the point, where the energy levels of the two wells coincide facilitating the tunneling. In the present latter we propose a different realization of the RR. Namely, the $\mathrm{RR}$ is observed as a function of the in-plane magnetic field (instead of the gate voltage). The maximal resistance corresponds to the zero magnetic field. The suppression of tunneling between two QWs in the parallel magnetic field was already demonstrated (in a different context) in Refs. [4] (perpendicular transport) and [5] (Shubnikov-de Haas oscillations). We employ the nice intuitive picture, developed in Refs. [4.5], to illustrate the results of our calculations. Below we present a microscopical description of the lateral magnetoresistance of the coupled QWs, which is verified by the experimental data. The main messages, following from our studies, are:

(i) The in-plane magnetic field destroys the coupling between QWs, leading to the RR; the lateral resistance is essentially anisotropic, means that the shape of the RR depends on the angle between the current and magnetic field. 
(ii) The width (i.e. the characteristic magnetic field, $H_{c}$ ) of the $\mathrm{RR}$ is sensitive to the single electron scattering time, providing a new method of measuring the small angle scattering time on the remote impurities.

(iii) The dependence of $H_{c}$ on temperature and on Fermi energy suggests that the electronelectron scattering rate (intralayer and interlayer) may be tested as well.

To develop a microscopical model of transport in two QWs we employ the basis of eigenstates of uncoupled wells. In this basis the Hamiltonian of the system is a $2 \times 2$ matrix, the off-diagonal elements of which represent the tunneling coupling between QWs

$$
\hat{H}_{\mathbf{k}, \mathbf{p}}=\delta_{\mathbf{k p}}\left(\begin{array}{cr}
\left(\mathbf{p}-e / c \mathbf{A}_{1}\right)^{2} /\left(2 m^{*}\right) & \Delta / 2 \\
\Delta / 2 & \left(\mathbf{p}-e / c \mathbf{A}_{2}\right)^{2} /\left(2 m^{*}\right)
\end{array}\right)+\left(\begin{array}{cc}
U_{1}(\mathbf{p}-\mathbf{k}) & 0 \\
0 & U_{2}(\mathbf{p}-\mathbf{k})
\end{array}\right) .
$$

We treat here only the case of coinciding quantized (in the $z$ direction) energy levels of the two wells. In Eq. (四) $-\mathbf{k}, \mathbf{p}$ are $2 \mathrm{D}$ momentum of the electrons, $\Delta$ is the tunneling gap (we assume tunneling to be momentum conserved) and $\mathbf{A}_{i}$ is a vector potential of an external field in the $i^{\text {th }}$ QW. The second matrix on the r.h.s. of Eq. (11) represents an elastic impurity scattering inside each QW. We assume that (1) random potentials $U_{i}(\mathbf{p}-\mathbf{k})$ have a finite correlation length in a plane of 2D gas, (2) there are no correlations between scatterers in different wells. In this case the disorder potential (in each well) may be described [6] by the single particle (small angle) mean free time, $\tau_{i}$, and the two particle (transport) mean free time, $\tau_{i}^{t r} \geq \tau_{i}$.

In a uniform magnetic field, $H$, parallel to the plane of the QW's (say directed along the $y$ direction) the corresponding vector potentials are $\mathbf{A}_{i}=\left(H z_{i}, 0\right)$, where $z_{i}$ are the $z$ coordinates of the effective centers of the QWs. The Fermi surfaces of two QWs have a form of two circles displaced along the $x$ direction (see Fig. 1) on the relative distance $k_{H}=e / c H b$, where $b=z_{1}-z_{2}$ is the distance between the centers of the wavefunctions in two wells [4, 5$]$. Only the electrons, which occupy the states in the vicinity of the (quasi)crossing points $A$ and $B$ (see Fig. 1), have the same energy $\left(\epsilon_{F}\right)$ and momentum in both wells and hence participate in the tunneling. Several important conclusions follow immediately:

(i) The resistance approaches its off-resonance value (decreases) as the magnetic field is 
increased.

(ii) The characteristic scale of the magnetic field may be estimated as $v_{F} k_{H} \approx \max \{\Delta, \hbar / \tau\}$ (see below).

(iii) The lateral resistance of the system in the in-plane magnetic field is anisotropic. Indeed, the transport in the $x$ direction is dominated by states with large $k_{x}$, which are practically decoupled (cf. Fig. 1 ). As a result the perpendicular (to the direction of the field) resistance is close to the off-resonance value. Contrary, the transport in the direction of the field is mostly determined by the states situated near the points $A$ and $B$ of Fig. 1 . These states are delocolized, making the parallel resistance closer to the resonance value. In other words, the suppression of the RR occurs in a different way depending on the angle between the magnetic field, $\mathbf{H}$, and the current, $\mathbf{j}$, used to probe the resistance.

The detailed diagrammatic calculation, based on the Kubo formula [7] leads to the following dependence of the resonance resistance on the in-plane magnetic field

$$
R^{-1}(H)-R_{o f f}^{-1}=\left(R^{-1}(0)-R_{o f f}^{-1}\right) f\left(H / H_{c}\right)
$$

where

$$
f(x)=\frac{2\left(\sqrt{1+x^{2}}-1\right)}{x^{2}} \begin{cases}1 ; & \mathbf{H} \| \mathbf{j} \\ \left(1+x^{2}\right)^{-1 / 2} ; & \mathbf{H} \perp \mathbf{j}\end{cases}
$$

and the characteristic field is given by

$$
H_{c}=\frac{\hbar c}{e} \frac{1}{v_{F} \tau b} \sqrt{1+\left(\frac{\Delta}{\hbar}\right)^{2} \frac{\tau_{1}^{t r}+\tau_{2}^{t r}}{2} \tau}
$$

finally $2 \tau^{-1} \equiv \tau_{1}^{-1}+\tau_{2}^{-1}$. The above relations are valid until $H \approx H_{F}$, where $H_{F} \equiv$ $2 \pi \hbar c /\left(e \lambda_{F} b\right) ; \lambda_{F}$ is a Fermi wavelength. Note also that $H_{c} \ll H_{F}$, as $\epsilon_{F} \tau / \hbar \gg 1$. In agreement with our expectation, the RR is suppressed faster in the perpendicular configuration, $R^{-1}(H)-R_{o f f}^{-1} \propto H^{-2}$, whereas in the parallel configuration $R^{-1}(H)-R_{\text {off }}^{-1} \propto H^{-1}$, for $H_{c} \ll H<H_{F}$.

The double QW structure was grown on $\mathrm{N}^{+}$GaAs substrate by molecular-beam epitaxy and consists of two GaAs wells $139 \stackrel{\circ}{A}$ width separated by a $40 \stackrel{\circ}{A} \mathrm{Al}_{0.3} \mathrm{Ga}_{0.7} \mathrm{As}$ barrier. The 
tunneling gap for this structure is estimated as $\Delta=0.55 \mathrm{meV}$. The electrons were provided by remote delta-doped donor layers set back by $250 \AA$ and $450 \AA$ spacer layers from the top and the bottom well correspondingly. In order to obtain the difference in the mobilities, an enhanced amount of impurities was introduced at the upper edge of the top well ( $\mathrm{Si}, 10^{10}$ $\mathrm{cm}^{-2}$ ). The schematic cross-section of the device may be found in Ref. [1]. Measurements were done on $10 \mu \mathrm{m}$-wide and $200 \mu \mathrm{m}$-long channels with $\mathrm{Au} / \mathrm{Ge} / \mathrm{Ni}$ Ohmic contacts. Top and bottom gates were patterned using the standard photolithography fabrication method. The top Schottky gate covered $150 \mu \mathrm{m}$ of the channel. The data were taken using a lockin four terminal techniques at $f=5.5 \mathrm{~Hz}$. The voltage probes connected to the gated segment of the channel were separated by $100 \mu \mathrm{m}$. The complementary measurements of the resistance and Hall coefficient leads to the following parameters of the structure (as grown, i.e., $V_{g}=V_{b g}=0$ and $\left.T=4.2 K\right): \mu_{1}=47,000 \mathrm{~cm}^{2} / V s e c, \mu_{2}=390,000 \mathrm{~cm}^{2} / V s e c, n_{1}=$ $4.7 \times 10^{11} \mathrm{~cm}^{-2}, n_{2}=2.5 \times 10^{11} \mathrm{~cm}^{-2}$. The values of these parameters for each temperature and gate voltage were determined independently and used for the fits (see below).

The variation of the top gate voltage, $V_{g}$ (for a fixed bottom gate voltage, $V_{b g}$ ), allows to sweep the potential profile of the QWs through the resonant configuration. The resistance vs. top gate voltage $\left(V_{b g}=1.5 \mathrm{~V} ; T=4.2 \mathrm{~K}\right)$ is plotted in the inset to Fig. 2 . The resistance resonance is clearly observed at $V_{g} \approx-0.2 \mathrm{~V}$. The value of the resistance in resonance is $R_{\text {res }}=65 \Omega$, whereas the off-resonance value is estimated as $R_{o f f}=32 \Omega$ (cf. Fig. 2, inset). Next we fix the gate voltges, corresponding to the exact resonance position, and measure the resistance as a function of the in-plane magnetic field. Fig. 2 shows the behavior of the $\mathrm{RR}$ for the two orientations of the magnetic field with respect to the direction of the current $(\mathbf{H} \| \mathbf{j}$ and $\mathbf{H} \perp \mathbf{j})$. The experimental data clearly demonstate the suppression of the RR by the magnetic field, as well as the expected anisotropy. In the perpendicular orientation the resistance decreases faster than in the parallel one. The theoretical curves, using Eqs. (2), (3) with $H_{c}=0.44 T$ (this is the only fitting parameter !) are shown on the same plot. For the perpendicular configuration, we obtained a perfect fit for the magnetic fields up to $H \approx 3 T$ (note that $H_{F} \approx 4.2 T$ for $\epsilon_{F}=15 \mathrm{meV}$ ). 
The situation is markedly different for the parallel configuration. The fit to the data is obtained only in the narrow range of fields up to $H_{c}$, at high magnetic fields the resistance does not approach the value $R_{o f f}=32 \Omega$. Moreover, a positive magnetoresistance contribution is well resolved. Large positive magnetoresistance $\left(\propto H^{2}\right)$ in the parallel configuration was also observed for the one QW (the second well was totally depleted by a large negative voltage on the top gate). We tend to attribute this positive contribution (hence the poor fit) to some normal to the plane of QWs component of the magnetic field, which is due to non perfect flatness of our structure in one direction. In the following, we thus restrict ourselves mostly on the perpendicular $(\mathbf{H} \perp \mathbf{j})$ orientation.

We employ now Eq. (4) and the extracted value of the characteristic field, $H_{c}=0.44 T$, to establish the small angle scattering time, $\tau$ (note that all other parameters entering Eq. (田) are known, see above). As a result one has $\hbar / \tau=1.7 \mathrm{meV}$ at $\mathrm{T}=4.2 \mathrm{~K}$, which implies the ratio between the transport and the small angle scattering times to be equal to $\approx 3.2$. Measurements of this ratio for different values of the Fermi energy (see below) result in a slow decrease from 3.2 at $\epsilon_{F}=15 \mathrm{meV}$ to 2.5 at $\epsilon_{F}=7 \mathrm{meV}$. These data are in a good agreement with the one measured, using Shubnikov-de Haas oscillations, in the 2D gas with the similar mobility [8]. It becomes evident now why one should complicate the theory to account the long range nature of scatterers. The simpler theory with short range scatterers only $\left(\tau_{i}^{t r}=\tau_{i}\right)$ fails to explain quantitatively the observed width of the RR. We conclude thus that the suppression of the RR in the magnetic field gives rise to a new and relatively simple way of measuring small angle scattering time.

We repeat then the same procedure (using $H_{c}$ as the only fitting parameter and then extracting $\tau$ ) for the perpendicular orientation at different temperatures in the range between $4.2 \div 40 K$. The experimental data and a set of theoretical curves is presented in Fig. 3 . The width of the curves increases with temperature indicating the increase of $H_{c}$. The same type of data were obtained for different set of voltages applied to the top and the bottom gates corresponding to the resonant conditions at different Fermi energies. The values of the Fermi energy in the range $7 \div 15 \mathrm{meV}$ were investigated. These data were also analyzed 
in the same fashion and the values of $\tau\left(T, \epsilon_{F}\right)$ were determined (we stress again that all relevant parameters, besides $\tau$, were established independently for each value of $T$ and $\epsilon_{F}$ ).

On the Fig. 4 we plot in a logarithmical scale $\epsilon_{F}(\hbar / \tau(T)-\hbar / \tau(0))$ versus temperature for three different values of the Fermi energy. At small enough temperature $\left(k_{B} T \ll \epsilon_{F}\right)$ all experimental points collapse to the same line. The slope of this line implies the quadratic temperature dependence of the displayed quantity. This way the following relation is established

$$
\frac{\hbar}{\tau(T)}-\frac{\hbar}{\tau(0)} \propto \frac{\left(k_{B} T\right)^{2}}{\epsilon_{F}}
$$

Eq. (5) suggests that the single particle scattering rate, $\tau^{-1}(T)$, consists of the two parts: small angle scattering rate on the remote impurities, $\tau^{-1}(0)$, and the electron-electron ( $\mathrm{e}-$ e) scattering rate, $\tau_{e e}^{-1}$. This is in contrast to the transport (two particle) scattering rate, $1 / \tau^{t r}$, which is practically not affected (in the clean limit, see below) by the e - e scattering (due to momentum conserved nature of the latest). To verify this idea quantitatively we use the result [9] for the $\mathrm{e}-\mathrm{e}$ scattering rate in a clean (the criterion is $\hbar / \tau(0) \ll k_{B} T \ll \epsilon_{F}$, which is fulfiled in our case) 2D gas

$$
\frac{\hbar}{\tau_{e e}}=(1+\xi) \frac{1}{\pi} \frac{\left(k_{B} T\right)^{2}}{\epsilon_{F}}\left(1+\ln 2+\ln \frac{\lambda_{F}}{\lambda_{T F}}-\ln \frac{k_{B} T}{\epsilon_{F}}\right),
$$

where $\lambda_{T F}=276 \AA$ is the Thomas-Fermi screening length in the GaAs. We have introduced in Eq. (6) an additional factor $(1+\xi)$, which intends to simulate intralayer and interlayer contributions to the $\mathrm{e}-\mathrm{e}$ scattering. In the original theory [9] only one 2D gas was considered thus $\xi \equiv 0$. In the case of two close QWs one expects that $0<\xi<1$, depending on the ratio between screening length, $\lambda_{T F}$, and the mean distance between the wells, $b$. This is indeed the case: the best fit to our data (the solid line in Fig. 4) is achieved by Eq. (6) with $\xi=0.5$. We conclude thus that in our structure the interlayer e - e scattering rate is 0.5 of the corresponding intralayer value. This seems to be reasonable since the distance between the wells is of the order of the screening length. To make more quantitative statements theory of $\mathrm{e}-\mathrm{e}$ interactions in two tunneling coupled QWs would be desirable. Eq. (6) is valid 
only in the limit $k_{B} T \ll \epsilon_{F}$. Therefore the deviations of the experimental points from the theory at high temperatures (especially for the smallest $\epsilon_{F}$ ) are not surprising. Our results may be considered as an other confirmation of the theory [9] in the range of relatively large temperatures. In a small temperature regime the theory [9] was excellently confirmed in the interference experiment [10].

The central point to all our discussions is the fact, that the transport quantity (the resistance) of the structure appears to be sensitive to the single particle scattering time (and not only to the transport one !). This enables us to determine the small angle scattering time, $\tau(0)$ (from the low temperature measurements, $k_{B} T<\hbar / \tau(0)$ ) as well as the $\mathrm{e}-\mathrm{e}$ scattering time, $\tau_{e e}$ (from the measurements at $k_{B} T>\hbar / \tau(0)$ ). The further comparison with the theory leads to the reasonable estimation of the ratio between intrawell and interwell e - e scattering rates. We believe thus that the RR in two coupled QWs with different mobilities provides a powerful and relatively simple method of measuring the small angle and $\mathrm{e}-\mathrm{e}$ scattering rates. Some unresolved questions raised in the present latter require further theoretical and experimental investigation of this phenomena.

We have benefited from the useful discussions with A. Aronov, O. Entin, V. Fleurov, Y. Gefen, D. Khmelnitskii, Y. Levinson and A. Yacoby. The experimental research was supported by Israel Academy of Sciences and Humanities. A.K. was supported by the German-Israel Foundation (GIF) and the U.S.-Israel Binational Science Foundation (BSF). 


\section{REFERENCES}

[1] A. Palevski, F. Beltram, F. Capasso, L. N. Pfeiffer and K. W. West, Phys. Rev. Lett. 65, 1929 (1990).

[2] A. Palevski, S. Luryi, P. L. Gammel, F. Capasso, L. N. Pfeiffer and K. W. West, Superlattices Microstruct.11, 269 (1992).

[3] Y. Ohno, M. Tsuchia and H. Sakaki, Appl. Phys. Lett.62, 1952 (1993).

[4] J. P. Eisenstein, T. J. Gramila, L. N. Pfeiffer, K. W. West, Phys. Rev.B44,6511 (1991)

[5] G. S. Boebinger, A. Passner, L. N. Pfeiffer, K. W. West, Phys. Rev. B 43, 12673 (1991).

[6] A. A. Abrikosov, L. P. Gorkov, and I. E. Dzyloshinski, Methods of Quantum Field Theory in Statistical Physics, Prentice-Hall, Inc., 1963.

[7] Y. Berk et al, to be published.

[8] P. T. Coleridge, Phys. Rev. B 44, 3793 (1991).

[9] G. F. Giuliani, and J. J. Quinn, Phys. Rev.B 26, 4421 (1982). Note the missed factor of two in the final result. For the discussion of this point see Ref. [10].

[10] A. Yacoby, M. Heiblum, H. Shtrikman, V. Umansky, and D. Mahalu, Semicon. Sci. Technol. 9, 907 (1994). 


\section{FIGURES}

Fig. 1. Fermi surfaces of two QWs in the in-plane magnetic field.

Fig. 2. The resonance resistance vs. magnetic field at $4.2 \mathrm{~K}$ : circles and asterisks experimental data for the perpendicular and parallel orientations correspondingly; solid and dashed lines - theoretical curves. The inset - lineshape of the RR vs. top gate voltage, $V_{g}$ $\left(V_{b g}=1.5 \mathrm{~V}\right)$.

Fig. 3. The resistance vs. magnetic field in the perpendicular configuration at different temperatures: circles - experiment; solid lines - theory $\left(V_{g b}=1.5 \mathrm{~V} ; V_{G}=-0.2 \mathrm{~V}\right.$, corresponding to $\epsilon_{F}=15 \mathrm{meV}$ ).

Fig. 4. Normalized scattering rate: $\frac{\epsilon_{F}}{11 m e V}\left(\frac{\hbar}{\tau(T)}-\frac{\hbar}{\tau(T)}\right)$ vs. temperature for different Fermi energies. The solid line: $\frac{\epsilon_{F}}{11 m e V} \frac{\hbar}{\tau_{e e}}$, where $\hbar / \tau_{e e}$ is given by Eq. (6) with $\xi=0.5$. 